\title{
Surgical treatment of dural arteriovenous malformation in the region of the sigmoid sinus
}

\author{
RUNE HUGOSSON AND KJELL BERGSTRÖM \\ From the Departments of Neurosurgery and Diagnostic Radiology, \\ University Hospital, Uppsala, Sweden
}

\begin{abstract}
SUMMARY The dural arteriovenous malformation in the occipitomastoid region usually has multiple arterial tributaries from the internal and external carotid and vertebral arteries. They converge towards the proximal segment of the sigmoid sinus, where the arterial blood is shunted into the venous channel. Five cases of this malformation are described. In four of them attempts were made to eliminate the symptoms by radical isolation of the transverse and sigmoid sinuses from all dural attachments. In addition, the mastoid bone over the fistular region had to be removed. To judge from our experience of these operations, this disease is curable.
\end{abstract}

Among the dural arteriovenous malformations in the posterior fossa of the skull, that in the occipitomastoid region is the most common, and from the pathologicoanatomical aspect it constitutes a well-defined entity. It may have a large number of afferent meningeal tributaries from the internal carotid, external carotid, and vertebral arteries, and the fistula is localized to the borderline between the transverse and sigmoid sinuses.

Several cases of this malformation have been described (Verbiest, 1961; Pecker et al., 1965; van Wijngarden and Vinken, 1966; Nicola and Nizzoli, 1968; Newton et al., 1968; Debrun and Chartres, 1972; Houser et al., 1972). Most of them were investigated thoroughly from an angiographic viewpoint but very little was achieved by surgical intervention because only one or two of the afferent arteries at the neck were attacked and ligated, which in most patients is quite inadequate.

There are two principal indications for operation. Firstly, the patients have an often disabling pulse-synchronous bruit in the head, causing insomnia and secondary mental disturbances. Secondly, there is a possible risk of hypoxic damage to the brain through the shunting of too much blood past the general circulation. During one year we have had five cases of this particular arteriovenous anomaly in our clinic. Attempts at radical surgical intervention have been made, and on the basis of our experiences from these cases the surgical technique of this radical operation is described here.

\section{RADIOLOGICAL FINDINGS}

Normally the occipital and posterior fossa region is vascularized by a large number of arteries (Salamon et al., 1971; Debrun and Chartres, 1972): (1) posterior branches of the middle meningeal artery; (2) posterior meningeal artery from the ascending pharyngeal artery; (3) meningeal branches of the posterior auricular artery; (4) meningeal branches of the occipital artery; (5) tentorial branches from the meningohypophyseal trunk of the carotid siphon; and (6) anterior and posterior meningeal branches of the vertebral artery.

Under pathological conditions when a dural arteriovenous malformation is present, many of the feeding arteries that normally are hardly visible on the arteriogram are hypertrophied. By the use of selective catheterization and the subtraction technique, these malformations can be described in detail radiologically, which is a basic prerequisite for successful surgical treatment. 


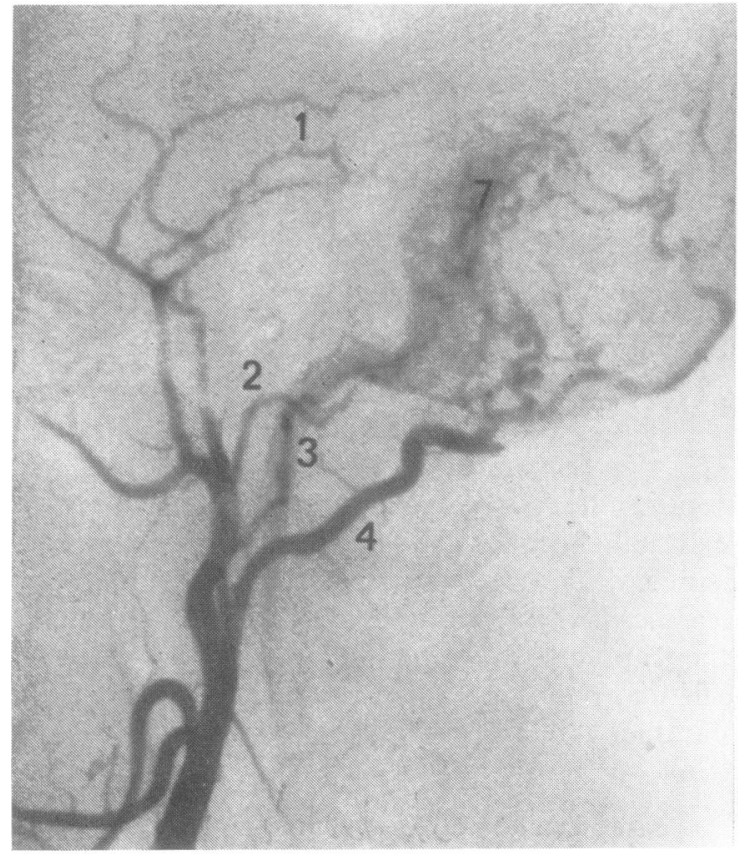

(a)

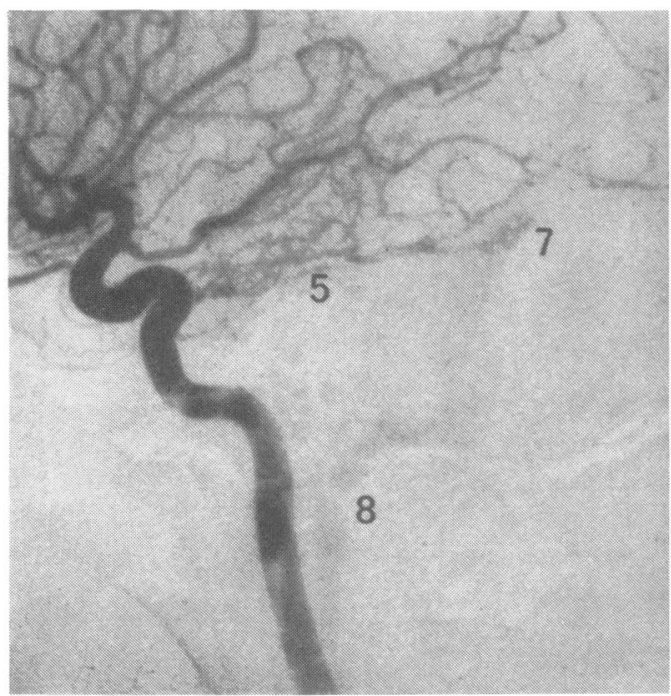

(c)

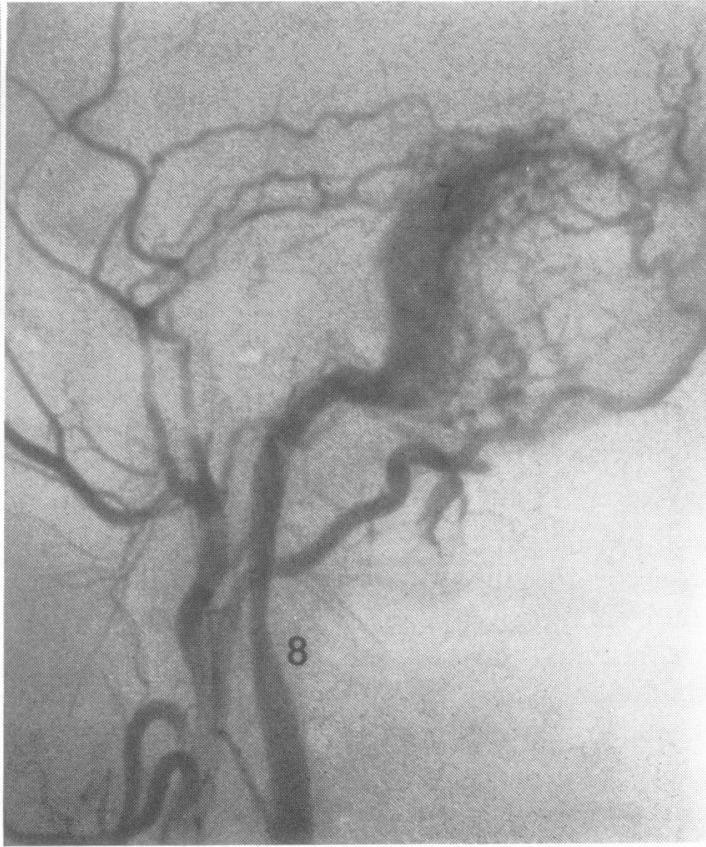

(b)

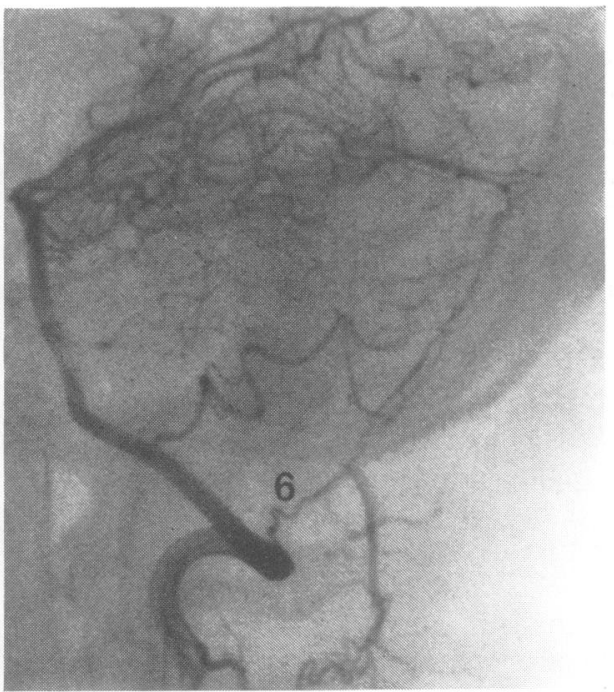

(d)

FIG. 1. Case 2. (a) and (b). Right external carotid arteriogram. (c) Right internal carotid arteriogram. (d) Right vertebral arteriogram. 1. Posterior branches of the middle meningeal artery. 2. Ascending pharyngeal artery. 3. Posterior auricular artery. 4. Occipital artery. 5. Tentorial branches of the internal carotid artery. 6. Posterior meningeal branch of the vertebral artery. 7. Drainage into the transverse-sigmoid sinus. 8. Internal jugular vein. 
TABLE

CONTRIBUTING ARTERIES TO ARTERIOVENOUS MALFORMATIONS IN TRANSVERSE-SIGMOID SINUS (FIVE CASES)

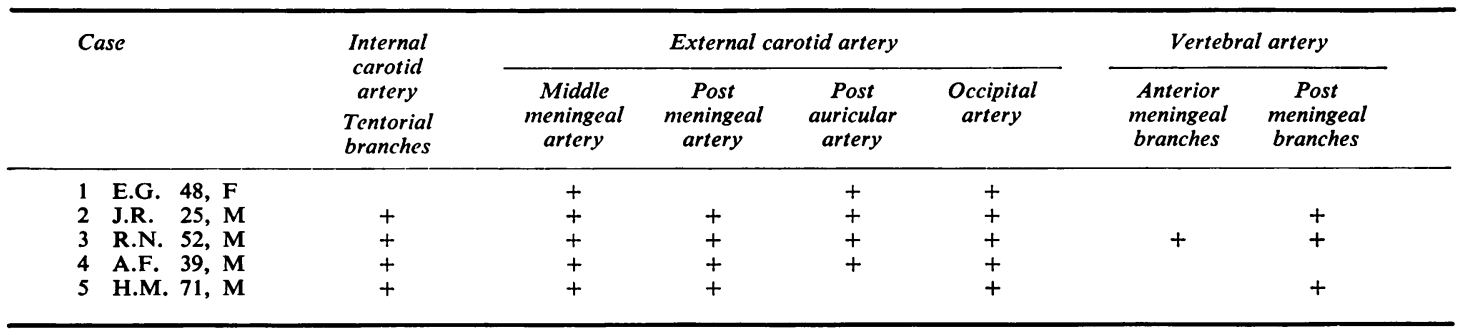

All of our five patients had multiple arteries leading to the fistula (Fig. 1 and the Table). Apart from the arteries listed in the Table, in case 2 there was also afferent flow from a distal branch of the posterior cerebral artery. In case 4 a posterior branch of the superficial temporal artery and muscular branches of the vertebral artery also supplied the fistula. It is probable, however, that these vessels did not proceed directly to the fistula but via anastomoses with

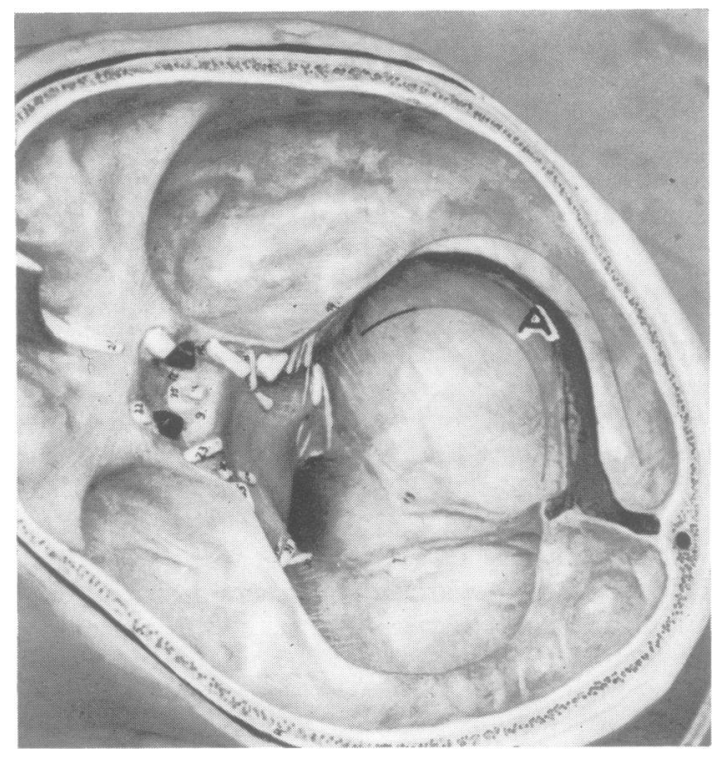

FIG. 2. Picture of the skull base with the tentorium removed and transverse-sigmoid sinuses opened. The curved lines indicate the dural incisions. Fistular region at $\mathrm{A}$. the occipital artery. In case 5, also, there was an unusual afferent supply-namely, from the ascending cervical artery. Normally, this artery gives off meningeal branches to the spinal dura mater in the cervical region, and these may possibly have established anastomoses with the intracranial meningeal vessels.

In all cases the venous drainage of all the afferent arteries took place within a limited area at the border between the transverse and sigmoid sinuses. There was no drainage into cortical veins.

\section{SURGICAL TECHNIQUE}

The principle of the operation is that the sinus segment in question must be isolated from the dura mater. This requires that a fairly large osteoplastic flap is turned over the transverse sinus, with wide exposure of both the occipital and the cerebellar dura mater. Underneath this flap, all afferent branches from the occipital artery are automatically ligated. Lumbar tapping of cerebrospinal fluid should be performed immediately before the operation as the occipital lobe is swollen due to impaired venous outflow.

Three long dural incisions are necessary (Fig. 2). The first is made in the occipital dura mater close to the midline and is continued above and parallel with the transverse sinus to the middle cranial fossa, ending at the superior petrosal sinus. Hereby all branches from the middle meningeal artery are divided. The second incision is made in the tentorium from the confluence in a lateral direction to the superior petrosal sinus. This sinus is now ligated and the two incisions meet. The bridging veins from the occipital lobe 
can cause technical difficulties and, in some cases, it is easier to cut through the tentorium from below. The third dural incision is made in the cerebellar dura mater the whole way along the transverse and sigmoid sinuses. It should continue, if possible, to the jugular foramen. This incision divides the meningeal branches from the vertebral artery and possibly also from the ascending pharyngeal artery. There remains now only a narrow dural stalk between the superior petrosal sinus and the sigmoid sinus, and this is most simply destroyed by coagulation.

When the dura mater has been opened in this way, it is time to expose the fistula part of the sinus. This is done by removal of the part of the mastoid bone that contains perforating vessels leading to the fistula from the posterior auricular artery and the occipital artery. The vascular channels in the bone can be occluded with bone wax and the openings in the sinus can be closed with fine silk sutures.

There is only one area of the dura mater that cannot be reached by this exploration and that is the outer wall of the basal portion of the sigmoid sinus. If the patient continues to hear the bruit postoperatively or if the malformation is still visible angiographically, the operation should be complemented with ligation of the ascending pharyngeal artery through a small incision on the neck.

\section{CASE REPORTS}

All five patients suffered from only one symptomnamely, a pulse-synchronous bruit; this was localized to the right ear in three patients and to the left ear in the other two. It was also common to all of them that the bruit increased in association with physical effort but was most troublesome during the night, when it caused insomnia. All patients had a hypertrophic occipital artery and three of them could make the bruit disappear by compression of this vessel. No neurological symptoms were detected in any of the patients. None of them had had any skull injury that could be related to the bruit.

The angiographic investigation comprised the internal and external carotid arteries and the vertebral artery, and in all cases showed an arteriovenous malformation in the occipitomastoid region. The afferent vessels in each individual case can be seen in the Table. The fistula area was located at the border between the transverse and sigmoid sinuses.
CASE 1 (E.G., 48 year old woman.) Operation 8 February 1972. The dura mater was opened above and below the transverse sinus so that the angiographically demonstrated meningeal vessels were divided. When the bone over the fistular region had been removed, the third afferent vessel could be occluded. Postoperatively, the bruit had completely disappeared and it has not recurred.

CASE 2 (J.R., 24 year old man.) Operation 15 February 1972. In this case only the occipital and temporal dura mater above the sinus were incised, and the bruit still persisted postoperatively. In a second session the occipital artery, the ascending pharyngeal artery, and the posterior auricular artery were therefore ligated at the neck. This had a good effect but in absolute silence the patient is still aware of a slight bruit. This emanates from the remaining tentorial branches and the meningeal branches of the vertebral artery.

CASE 3 (R.N., 52 year old man.) Operation 25 April 1972. In this patient the operation was performed according to the principles described under Surgical technique, except that the superior petrosal sinus was not divided. Apart from a dysphasia which very quickly regressed, the postoperative course was uneventful. The bruit disappeared completely and has not recurred.

CASE 4 (A.F., 39 year old man.) Operation 11 November 1972. This patient underwent exactly the same operation as case 3 , and the bruit disappeared. The operation was complicated by a subdural hygroma which was evacuated, and a wound infection, but gradually the patient recovered completely.

Postoperative angiography in the common carotid artery revealed, however, that the sigmoid sinus still filled with contrast medium via one of the tentorial branches and the ascending pharyngeal artery. Some weeks after the operation the patient also stated that in absolute silence he could hear a very weak sound in his right ear.

A second session with ligation of the ascending pharyngeal artery always has to be reckoned with in these cases. As regards the intracranial operation, our experience from case 4 shows that the division of the tentorium must include the petrosal sinus, as some of the tentorial branches may run in the wall of this sinus on their way to the fistula.

CASE 5 (H.M., 71 year old man.) No attempt at a radical operation was made in this patient, on account of his age. The occipital artery was ligated but this had no effect on the bruit. 


\section{DISCUSSION}

Verbiest (1951) was the first to describe a dural arteriovenous malformation in the posterior fossa with an attempt at surgical treatment. The malformation was localized to the tentorium. Later, however (1962), he reported a case that was very similar to ours. The operation consisted of ligation of the external carotid and occipital arteries, but the bruit still persisted. Since this date, completely or almost identical cases have been described by Pecker et al. (1965, one case), Wijngaarden and Vinken (1966, one case), Newton et al. (1968, eight cases), Debrun and Chartres (1972, one case), and Houser et al. (1972, 11 cases). In the patients who underwent operation, this consisted of ligation of one or two of the afferent vessels at the neck. These operations were not successful in eliminating the bruit, however; Pecker's case has been described as a success but the patient still has tentorial and meningeal branches of the vertebral artery feeding the shunt. Among the cases described by Nicola and Nizzoli (1968) there is one similar to ours. In this patient the occipital and suboccipital regions were explored and the largest vessels supplying the malformation were ligated. No information was given concerning the bruit, however.

Surgical treatment consisting of ligation of afferent vessels at the neck is not sufficient. In most cases the malformation is also supplied by tributaries from the carotid siphon and the vertebral artery. These gradually become hypertrophied and the symptoms return. A more extensive operation aiming at the most complete possible isolation of the transverse and sigmoid sinuses is therefore necessary. This is done by cutting all dural communications with the sinuses and extirpation of the bone covering the fistular region. In two of our patients our operation was not sufficiently radical, but to judge from our over-all experiences from this small series these lesions should be curable.

Only one particular and well-defined arteriovenous malformation has been described in this paper, but in principle this type of surgical approach should also be valid for other dural vascular anomalies in the posterior part of the cranium.

\section{REFERENCES}

Debrun, G., and Chartres, A. (1972). Infra and supratentorial arteriovenous malformations. A general review. Neuroradiology, 3, 184-192.

Houser, O. W., Baker, H. L., Jr., Rhoton, A. L., Jr., and Okazaki, H. (1972). Intracranial dural arteriovenous malformations. Radiology, 105, 55-64.

Newton, T. H., Weidner, W., and Greitz, T. (1968). Dural arteriovenous malformation in the posterior fossa. Radiology, 90, 27-35.

Nicola, G. C., and Nizzoli, V. (1968). Dural arteriovenous malformations of the posterior fossa. Journal of Neurology, Neurosurgery, and Psychiatry, 31, 514-519.

Pecker, J., Bonnal, J., and Javalet, A. (1965). Deux nouveaux cas d'anévrysmes artério-veineux intraduraux de la fosse postérieure alimentés par la carotide externe. Neurochirurgie, 11, 327-332.

Salamon, G. M., Combalbert, A., Raybaud, C., and Gonzalez, J. (1971). An angiographic study of meningiomas of the posterior fossa. Journal of Neurosurgery, 35, 731-741.

Verbiest, H. (1951). L'anévrisme artérioveineux intradural. Revue Neurologique, 85, 189-199.

Verbiest, H. (1962). Arterial and arteriovenous aneurysms of the posterior fossa. Psychiatria, Neurologia, Neurochirurgia, 65, 329-369.

Wijngaarden, G. K. van, and Vinken, P. J. (1966). A case of intradural arteriovenous aneurysm of the posterior fossa. Neurology (Minneap.), 16, 754-756. 